\title{
Assessment of genetic diversity, population structure and relationships in Indian and non-Indian genotypes of finger millet (Eleusine coracana (L.) Gaertn) using genomic SSR markers
}

\author{
M. Ramakrishnan', S. Antony Ceasar ${ }^{1,2}$, V. Duraipandiyan ${ }^{1,3}$, N. A. Al-Dhabi ${ }^{3}$ and S. Ignacimuthu ${ }^{1,4^{*}}$
}

\begin{abstract}
We evaluated the genetic variation and population structure in Indian and non-Indian genotypes of finger millet using 87 genomic SSR primers. The 128 finger millet genotypes were collected and genomic DNA was isolated. Eighty-seven genomic SSR primers with 60-70 \% GC contents were used for PCR analysis of 128 finger millet genotypes. The PCR products were separated and visualized on a $6 \%$ polyacrylamide gel followed by silver staining. The data were used to estimate major allele frequency using Power Marker v3.0. Dendrograms were constructed based on the Jaccard's similarity coefficient. Statistical fitness and population structure analyses were performed to find the genetic diversity. The mean major allele frequency was 0.92; the means of polymorphic alleles were 2.13 per primer and 1.45 per genotype; the average polymorphism was $59.94 \%$ per primer and average PIC value was 0.44 per primer. Indian genotypes produced an additional 0.21 allele than non-Indian genotypes. Gene diversity was in the range from 0.02 to 0.35 . The average heterozygosity was 0.11 , close to $100 \%$ homozygosity. The highest inbreeding coefficient was observed with SSR marker UGEP67. The Jaccard's similarity coefficient value ranged from 0.011 to 0.836 . The highest similarity value was 0.836 between genotypes DPI009-04 and GPU-45. Indian genotypes were placed in Eleusine coracana major cluster (ECMC) 1 along with 6 non-Indian genotypes. AMOVA showed that molecular variance in genotypes from various geographical regions was $4 \%$; among populations it was $3 \%$ and within populations it was $93 \%$. PCA scatter plot analysis showed that GPU-28, GPU-45 and DPI009-04 were closely dispersed in first component axis. In structural analysis, the genotypes were divided into three subpopulations (SP1, SP2 and SP3). All the three subpopulations had an admixture of alleles and no pure line was observed. These analyses confirmed that all the genotypes were genetically diverse and had been grouped based on their geographic regions.
\end{abstract}

Keywords: Finger millet, Genetic diversity, AMOVA, PCA, Population structure, Baysian statistics

\section{Background}

Micronutrient deficiency has been recognized as a serious human health problem worldwide (Kanatti et al. 2014). Finger millet (Eleusine coracana L. Gaertn.) is a highly self-pollinating crop majorly cultivated in less

\footnotetext{
*Correspondence: eriloyola@hotmail.com

1 Division of Plant Biotechnology, Entomology Research Institute, Loyola

College, Chennai 600 034, India

Full list of author information is available at the end of the article
}

developed countries of Asia and Africa. It is a good source of micronutrients like, iron and zinc. Biofortification of staple crops is a sustainable and cost-effective approach for availability of micronutrients. Biofortified cultivars of finger millet for improved micronutrients are acceptable to consumers as their adoption does not call for change in dietary habits. Analysis of genetic diversity leading to molecular breeding is a major approach for development of biofortified cultivars of finger millet.

\section{望 Springer}


Study of genetic diversity and population structure between genotypes has long been a major goal for crop development (Qin et al. 2009; Yang et al. 2013; Egbadzor et al. 2014; Sharma et al. 2014). India is the largest producer of finger millet and more than 34,160 cultivable genotypes are available world-wide and India alone has 22,583 genotypes; these include 9522 genotypes with National Bureau of Plant Genetic Resources (NBPGR), New Delhi, 6804 genotypes with International Crops Research Institute for the Semi-Arid Tropics (ICRISAT), Patancheru, Hyderabad and 6257 genotypes with All India Coordinated Minor Millet Project (AICMMP), Bangalore (Goron and Raizada 2015). However, only few authors have reported the genetic diversity analysis of finger millet genotypes using simple sequence repeats (SSR) markers. The numbers of genotypes used by various groups for genetic diversity analysis of finger millet genotypes using SSR markers include 79 by Dida et al. (2008), 83 by Panwar et al. (2010b), 52 by Panwar et al. (2010a) and Kumar et al. (2012), 67 by Arya et al. (2013), 103 by Nirgude et al. (2014) and 190 by Babu et al. (2014b).

SSR are tandem repeats of 2-6 base pairs which are highly polymorphic and variable in the number of repeats at a specific locus. They are widely distributed throughout the genomes in both coding and non-coding regions; they are codominant, multi-allelic, chromosome specific and highly informative genetic markers (Cho et al. 2000; Scott et al. 2000). They are amenable to high throughput genotyping, thus suitable for diversity analysis (Hua et al. 2015; Wang et al. 2011). The SSR markers have been used successfully for the evaluation of genetic diversity among several species, including finger millet (Babu et al. 2014b), foxtail millet (Kim et al. 2012; Zhao et al. 2012) and proso millet (Cho et al. 2010).

The present study aimed to assess the extent of genetic variation and population structure at the molecular level in 64 Indian, 61 non-Indian and 3 unknown origin finger millet genotypes with a view to provide data for breeding programes. In the present study, 87 genomic SSR primers were used to study the genetic variation and population structure among 128 genotypes of finger millet. Data generated through this study will be useful for breeding programes and as a resource for gene banks in future to improve the finger millet genotypes.

\section{Methods}

\section{Plant materials and isolation of genomic DNA}

The details of 128 finger millet genotypes and their origins are listed in Table 1. Seeds of these genotypes were obtained from the ICRISAT, Patancheru, India, University of Agricultural Sciences, Bangalore, India and Tamil Nadu Agricultural University, Coimbatore, India.
The genomic DNA was isolated from all 128 genotypes (3 plants for each genotype) based on the protocol described in our previous report (Ramakrishnan et al. 2015). The purity and concentration of DNA were quantified using a Nanodrop-spectrophotometer (ND2000,ThermoScientific, Wilmington, DE, USA) and the DNA was diluted to $50 \mathrm{ng} / \mu \mathrm{l}$ concentration with $0.1 \times \mathrm{TE}$ buffer for SSR genotyping.

\section{PCR amplification and silver staining}

Eighty-seven genomic SSR primers with 60-70 \% GC contents were used to study the genetic diversity. The PCR reactions were performed in $25 \mu \mathrm{l}$ reaction mixture containing $50 \mathrm{ng}$ each of genomic DNA, $2.5 \mathrm{mM} \mathrm{MgCl}$, $0.25 \mathrm{mM}$ dNTPs, $400 \mathrm{nM}$ each of forward and reverse primers and 1 U Taq DNA Polymerase (Genet Bio, Daejeon, Korea). The amplification was carried out in a DNA thermal cycler (Eppendorf, Gradient Thermal Cycler, Germany). The PCR was performed with an initial denaturation at $95{ }^{\circ} \mathrm{C}$ for $5 \mathrm{~min}$ followed by 35 cycles of $30 \mathrm{~s}$ denaturation at $95{ }^{\circ} \mathrm{C}, 30 \mathrm{~s}$ annealing at different temperatures based on the primer pair and 1 min extension at $72{ }^{\circ} \mathrm{C}$ with a final extension at $72{ }^{\circ} \mathrm{C}$ for $10 \mathrm{~min}$. The PCR products were separated and visualized on a $6 \%$ polyacrylamide gel followed by silver staining. The fragment sizes of the PCR products were estimated by comparison with 100 base pair (bp) and 50 bp DNA ladders; alleles size were visually scored in bp for each genotype; amplification was repeated with each primer to confirm the reproducibility of the results.

\section{Genetic statistics}

The data were used to estimate major allele frequency, allele number, gene diversity, heterozygosity, polymorphic information content (PIC) and inbreeding coefficient using Power Marker v3.0 (Liu and Muse 2005). Dendrograms were constructed with 1000 bootstrapping values using unweighted pair-group method with arithmetic average (UPGMA) based on the Jaccard's similarity coefficient (Jaccard 1908) using FREE TREE and TREE VIEW softwares. Analysis of the molecular variation (AMOVA) was performed using GenALEx software version 6.5 (Peakall and Smouse 2012) to test the total molecular variance among the various geographical regions, among the populations and within the populations.

\section{Statistical fitness analysis}

To validate the cluster analysis and genetic structure, the cophenetic correlation coefficient (CCC) value was calculated using UPGMA. The distribution of populations was analyzed using Principal component analysis (PCA) which was carried out using PAST version 2 software 
Table 1 Details of finger millet genotypes collected from different geographical regions used for the analysis of genetic diversity using genomic SSR markers

\begin{tabular}{|c|c|c|c|c|c|c|c|}
\hline Varieties & $\begin{array}{l}\text { Source } \\
\text { country }\end{array}$ & Varieties & $\begin{array}{l}\text { Source } \\
\text { country }\end{array}$ & Varieties & $\begin{array}{l}\text { Source } \\
\text { country }\end{array}$ & Varieties & $\begin{array}{l}\text { Source } \\
\text { country }\end{array}$ \\
\hline APSKK-1 & India & SVK-1 & India & IE-2957 & Germany & IE-4795 & Zimbabwe \\
\hline CO- (RA) 14 & India & $\mathrm{T}-\mathrm{CHIN}-1$ & India & IE-3045 & India & IE-4797 & Maldives \\
\hline CO-(NO)-1 & India & T-CUM-1 & India & IE-3077 & India & IE-4816 & India \\
\hline $\mathrm{CO}-11$ & India & THRV-P & India & IE-3104 & India & IE-5066 & Senegal \\
\hline $\mathrm{CO}-12$ & India & THRV-PP & India & IE-3317 & Zimbabwe & IE-5091 & Zimbabwe \\
\hline $\mathrm{CO}-7$ & India & TRY-1 & India & IE-3391 & Zimbabwe & IE-5106 & Zimbabwe \\
\hline $\mathrm{CO}-9$ & India & VIJAYAWADA & India & IE-3392 & Zimbabwe & IE-5201 & India \\
\hline GPU-26 & India & $V L-149$ & India & IE-3470 & India & IE-5306 & Zimbabwe \\
\hline GPU-28 & India & VR-708 & India & IE-3475 & India & IE-5367 & Kenya \\
\hline GPU-45 & India & THRP-1 & India & IE-3614 & NA & IE-5537 & Nepal \\
\hline GPU-46 & India & IE-501 & India & IE-3618 & NA & IE-5817 & Nepal \\
\hline GPU-48 & India & IE-518 & India & IE-3721 & Uganda & IE-5870 & Nepal \\
\hline GPU-66 & India & IE-1055 & NA & IE-3945 & Uganda & IE-6059 & Nepal \\
\hline GPU-67 & India & IE-2034 & India & IE-3952 & Uganda & IE-6082 & Nepal \\
\hline HOSUR-1 & India & IE-2042 & India & IE-3973 & Uganda & IE-6154 & Nepal \\
\hline HR-374 & India & IE-2043 & India & IE-4028 & Uganda & IE-6165 & Nepal \\
\hline HR-911 & India & IE-2217 & India & IE-4057 & Uganda & IE-6221 & Nepal \\
\hline INDOF-5 & India & IE-2296 & India & IE-4073 & Uganda & IE-6240 & Zimbabwe \\
\hline INDOF-7 & India & IE-2312 & India & $\mid E-4121$ & Uganda & IE-6294 & Zimbabwe \\
\hline INDOF-8 & India & IE-2430 & Kenya & IE-4329 & Zimbabwe & IE-6326 & Zimbabwe \\
\hline INDOF-9 & India & IE-2437 & Kenya & $\mid \mathrm{E}-4491$ & Zimbabwe & IE-6337 & Zimbabwe \\
\hline KM-252 & India & IE-2457 & Kenya & IE-4497 & Zimbabwe & IE-6350 & Zimbabwe \\
\hline KMR-301 & India & $\mathrm{IE}-2572$ & Kenya & IE-4545 & Zimbabwe & IE-6421 & Uganda \\
\hline$\llcorner-5$ & India & IE-2589 & USA & IE-4565 & Zimbabwe & IE-6473 & Uganda \\
\hline M6-6 & India & IE-2606 & Malawi & $\mathrm{IE}-4570$ & Zimbabwe & IE-6514 & Zimbabwe \\
\hline$M L-365$ & India & IE-2619 & Malawi & $\mid E-4622$ & Zimbabwe & IE-6537 & Nigeria \\
\hline MR-1 & India & $\mathrm{IE}-2710$ & Malawi & $\mathrm{IE}-4646$ & Zimbabwe & IE-7018 & Kenya \\
\hline MR-2 & India & IE-2790 & Malawi & $\mathrm{IE}-4671$ & India & IE-7079 & Kenya \\
\hline PAIYUR-2 & India & IE-2821 & Nepal & IE-4673 & India & IE-7320 & Kenya \\
\hline PES-110 & India & IE-2871 & Zambia & IE-4709 & Burundi & KRI007-01 & India \\
\hline PR-202 & India & IE-2872 & Zambia & IE-4734 & India & DPI009-04 & India \\
\hline RAU-8 & India & IE-2911 & Zambia & IE-4757 & India & KRI13-11 & India \\
\hline
\end{tabular}

(Hammer et al. 2001). The number of significant components to interpret from PCA was determined by both Jolliffe cut-off value and broken stick model (Jolliffe 2002).

\section{Population structure analysis}

Analysis of the population structure and gene flow between 128 finger millet genotypes was carried out with 87 genomic SSR primers using a model-based Bayesian statistics implemented to subdivide genotypes into genetic subpopulations (SPs) using the software STRUCTURE v.2.3.4 (Pritchard et al. 2000; Ramasamy et al. 2014). In the present study, no prior knowledge was used to define SP and it was expected that number of SPs existed in the sample analyzed. Each genotype can be a member of a different subgroup (admixture model; ALPHAPROPSD $=0.20$ ). The number of subgroups $(K)$ in the population was determined by running the programme with $K$ values varying from 1 to 10 , with five independent runs for each $K$ value. To determine most appropriate $K$ value, burn-in Markov Chain Monte Carlo (MCMC) (Bayesian statistics) (Karandikar 2006) replication was set to 100,000 and data were collected over 500,000 MCMC replications in each run. The $K$ value was detected using (Structure Harvester) an ad hoc statistic $\Delta K$ based on the rate of change in the log probability of data between successive $K$ values (Evanno et al. 2005). 


\section{Results}

\section{Statistical analysis of genomic SSR markers}

The number of scorable alleles produced per primer ranged from 1 to 7 . Primer SSR02 generated highest number of alleles of 7 and primers UGEP84, UGEP102 and UGEP109 generated least number of allele of 1 . The major allele frequency of SSR markers ranged from 0.80 to 0.99 and mean major allele frequency was 0.92 . The UGEP84 showed highest major allele frequency of 0.99, while SSR01 showed lowest major allele frequency of 0.80 . Totally 252 alleles were produced, of which 186 (73.80 \%) were polymorphic with an average of 2.13 alleles per primer and 1.45 alleles per genotype. Out of 87 markers, 72 (82.75\%) were found to be polymorphic. Among polymorphic markers the percentage of polymorphism ranged from 25.0 to $85.71 \%$; average polymorphism was $59.94 \%$ per marker. Primer SSR10 produced highest polymorphism of $85.71 \%$ and primer UGEP69 produced lowest polymorphism of $25 \%$. The polymorphic alleles were informative to differentiate the selected genotypes. In Indian genotypes, total number of alleles was 136 with an average of 1.5 alleles per primer and 1.06 alleles per genotype. In non-Indian genotypes, the total number of alleles was 110 with an average of 1.26 alleles per primer and 0.85 allele per genotype. In three unknown genotypes, the total number of alleles was 6 with an average of 2 alleles per genotype. Indian genotypes produced an additional 0.21 allele than non-Indian genotypes.

Gene diversity was in the range of $0.02-0.35$ with an average value of 0.14 and gene diversity was found to be highest with the primer SSR01 (0.35), followed by SSR02 and SSR10 (0.33). Forty SSR primers showed more gene diversity than the average value (0.14). The heterozygosity ranged from 0.0 to 0.26 and SSR10 showed highest heterozygosity (0.26), followed by UGEP3 (0.25); average heterozygosity was 0.11 , close to $100 \%$ homozygosity. The PIC values ranged from 0.32 to 0.64 ; the average PIC value was 0.44 . Primer SSR01 produced highest PIC value of 0.64 and primers UGEP20, UGEP27, UGEP58, UGEP66, UGEP70, UGEP74 and UGEP84 produced lowest PIC value of 0.32 . The inbreeding coefficient value ranged from 0.0 to 1.0 and the average value was 0.34 . The highest inbreeding coefficient value was observed with UGEP67, UGEP84 and UGEP87 (1); this confirmed heterozygosity. The SSRs which had heterozygosity value of 0 showed highest inbreeding coefficient value of 1 (Table 2).

\section{Jaccard's similarity coefficient}

The value of Jaccard's similarity coefficients ranged from 0.011 to 0.836 . In UPGMA cluster analysis, the genotypes were grouped into three major clusters viz,
Eleusine coracana major cluster (EcMC) 1 to EcMC3. Indian genotypes DPI009-04 and GPU-45 were placed in EcMC1. The value of Jaccard's similarity coefficients was 0.836 between these 2 genotypes; this was the highest similarity value obtained between genotypes in this study whereas the lowest value of 0.011 was observed between genotypes IE-3392 and IE-3470, IE-6221 and IE-6240, IE-4073 and IE-4121, and IE-6165 and IE-6221. Between genotypes IE-2437 and IE-2457 the value was 0.019 . The similarity value was 0.773 between Indian genotypes GPU-28 and GPU-45. These genotypes (GPU 28 and GPU-45) are blast resistant local varieties cultivated in Karnataka state, India. The Indian genotypes GPU26, GPU-28, GPU-45, KRI007-01, KRI1311 and GPU-67 were placed in EcMC1 along with IE-7079 which originated from Kenya.

Blast susceptible genotypes RAU-8 and CO-9 were placed in EcMC1 and the value of Jaccard's similarity coefficients between them was 0.449. Another blast susceptible genotype KM-252 was also clustered in EcMC1 with high yielding genotype Paiyur-2; their Jaccard's similarity coefficients value was 0.547 . Out of 64 Indian genotypes, 56 genotypes were placed in EcMC1 along with 6 non-Indian genotypes, IE-2430 and IE-7079 (Kenya), IE-2790 (Malawi), IE-2957 (Germany), IE-3721 (Uganda) and IE-6514 (Zimbabwe) (Fig. 1). This may be due to the fact that the Indian genotypes might have originated from Kenya and Zimbabwe.

The $78.08 \%$ of clusters showed higher bootstrap values with $70-100 \%$ and only $21.91 \%$ of clusters showed lower bootstrap values with 40-69\%. These values confirmed that cluster nodes were well supported and none of the cluster node was found to be poorly supported. Indian genotypes, APSSK-1 and CO-12, THRV-PP and TCUM1 , and CO-14 and TRY-1 showed higher bootstrap values (100\%). Likewise 10 non-Indian genotypes, IE-4570, IE-2872, IE-4795, IE-4709, IE-6059, IE-5066, IE-5817, IE-3945, IE-6240 and IE-3392 showed higher bootstrap values $(100 \%)$. Based on the cluster grouping and the bootstrap value (100\%) it has been confirmed that all 64 Indian genotypes are genetically distinct from 61 nonIndian genotypes.

\section{Statistical fitness analysis}

PCA analysis showed that first and third component axes accounted for 12.08 and $3.21 \%$ respectively of the total variance and eigenvalues were 5.04 and 1.3 respectively. PCA plot was made using the first and third components based on the Var-covar matrix which showed that Indian genotypes GPU-28, GPU-45 and DPI009-04 were closely dispersed in first component axis (Fig. 2). The non-Indian genotype IE-2790 from Malawi has dispersed distantly in third coordinate with genotypes IE-3475 and IE-4673 of 
Table 2 List of 87 genomic SSR primers with polymorphism details, used for the analysis of genetic diversity and population structure of 128 finger millet genotypes collected from various geographical regions of the world

\begin{tabular}{|c|c|c|c|c|c|c|c|c|c|c|c|c|c|}
\hline Marker & MAF & AN & GD & $\mathrm{He}$ & PIC & IC & Marker & MAF & AN & GD & $\mathrm{He}$ & PIC & IC \\
\hline SSR01 & 0.80 & 5.0 & 0.35 & 0.23 & 0.64 & 0.33 & UGEP68 & 0.90 & 2.0 & 0.18 & 0.15 & 0.48 & 0.19 \\
\hline SSR02 & 0.82 & 7.0 & 0.33 & 0.20 & 0.62 & 0.41 & UGEP69 & 0.98 & 2.0 & 0.05 & 0.03 & 0.35 & 0.33 \\
\hline SSR06 & 0.84 & 3.0 & 0.29 & 0.23 & 0.59 & 0.23 & UGEP70 & 0.99 & 3.0 & 0.02 & 0.01 & 0.32 & 0.67 \\
\hline SSR08 & 0.85 & 6.0 & 0.27 & 0.19 & 0.56 & 0.31 & UGEP73 & 0.97 & 3.0 & 0.05 & 0.04 & 0.35 & 0.28 \\
\hline SSR10 & 0.81 & 4.0 & 0.33 & 0.26 & 0.62 & 0.23 & UGEP74 & 0.99 & 2.0 & 0.02 & 0.01 & 0.32 & 0.67 \\
\hline UGEP1 & 0.84 & 3.0 & 0.30 & 0.22 & 0.59 & 0.26 & UGEP75 & 0.95 & 4.0 & 0.11 & 0.05 & 0.40 & 0.56 \\
\hline UGEP3 & 0.82 & 3.0 & 0.32 & 0.25 & 0.61 & 0.22 & UGEP76 & 0.87 & 2.0 & 0.24 & 0.16 & 0.54 & 0.33 \\
\hline UGEP5 & 0.83 & 2.0 & 0.31 & 0.23 & 0.60 & 0.24 & UGEP77 & 0.88 & 2.0 & 0.22 & 0.13 & 0.52 & 0.41 \\
\hline UGEP6 & 0.84 & 3.0 & 0.28 & 0.23 & 0.57 & 0.20 & UGEP78 & 0.86 & 3.0 & 0.26 & 0.19 & 0.55 & 0.27 \\
\hline UGEP7 & 0.88 & 3.0 & 0.22 & 0.19 & 0.51 & 0.14 & UGEP79 & 0.98 & 3.0 & 0.03 & 0.02 & 0.33 & 0.50 \\
\hline UGEP8 & 0.86 & 3.0 & 0.25 & 0.16 & 0.54 & 0.34 & UGEP80 & 0.98 & 4.0 & 0.04 & 0.02 & 0.34 & 0.40 \\
\hline UGEP9 & 0.93 & 4.0 & 0.14 & 0.12 & 0.44 & 0.17 & UGEP81 & 0.91 & 2.0 & 0.18 & 0.17 & 0.47 & 0.02 \\
\hline UGEP10 & 0.84 & 2.0 & 0.28 & 0.20 & 0.57 & 0.28 & UGEP83 & 0.96 & 3.0 & 0.08 & 0.04 & 0.38 & 0.54 \\
\hline UGEP11 & 0.88 & 3.0 & 0.22 & 0.17 & 0.51 & 0.21 & UGEP84 & 0.99 & 1.0 & 0.02 & 0.00 & 0.32 & 1.00 \\
\hline UGEP12 & 0.86 & 2.0 & 0.26 & 0.22 & 0.55 & 0.15 & UGEP86 & 0.98 & 2.0 & 0.04 & 0.02 & 0.34 & 0.40 \\
\hline UGEP13 & 0.93 & 5.0 & 0.14 & 0.12 & 0.44 & 0.18 & UGEP87 & 0.98 & 3.0 & 0.03 & 0.00 & 0.33 & 1.00 \\
\hline UGEP15 & 0.88 & 3.0 & 0.22 & 0.16 & 0.51 & 0.28 & UGEP88 & 0.98 & 3.0 & 0.03 & 0.02 & 0.33 & 0.50 \\
\hline UGEP16 & 0.90 & 3.0 & 0.18 & 0.10 & 0.48 & 0.45 & UGEP90 & 0.88 & 2.0 & 0.21 & 0.20 & 0.50 & 0.09 \\
\hline UGEP17 & 0.93 & 3.0 & 0.13 & 0.08 & 0.43 & 0.42 & UGEP91 & 0.96 & 2.0 & 0.07 & 0.02 & 0.37 & 0.66 \\
\hline UGEP18 & 0.86 & 4.0 & 0.26 & 0.21 & 0.55 & 0.20 & UGEP93 & 0.97 & 3.0 & 0.06 & 0.05 & 0.36 & 0.23 \\
\hline UGEP19 & 0.90 & 3.0 & 0.19 & 0.14 & 0.48 & 0.26 & UGEP95 & 0.95 & 3.0 & 0.11 & 0.09 & 0.40 & 0.11 \\
\hline UGEP20 & 0.99 & 3.0 & 0.02 & 0.01 & 0.32 & 0.67 & UGEP96 & 0.95 & 3.0 & 0.09 & 0.05 & 0.39 & 0.49 \\
\hline UGEP21 & 0.89 & 2.0 & 0.20 & 0.15 & 0.49 & 0.25 & UGEP97 & 0.96 & 2.0 & 0.08 & 0.05 & 0.38 & 0.34 \\
\hline UGEP22 & 0.97 & 3.0 & 0.05 & 0.04 & 0.35 & 0.27 & UGEP98 & 0.98 & 3.0 & 0.04 & 0.02 & 0.34 & 0.40 \\
\hline UGEP24 & 0.86 & 2.0 & 0.26 & 0.24 & 0.55 & 0.08 & UGEP100 & 0.96 & 3.0 & 0.07 & 0.04 & 0.37 & 0.44 \\
\hline UGEP25 & 0.98 & 3.0 & 0.04 & 0.02 & 0.34 & 0.39 & UGEP101 & 0.97 & 4.0 & 0.06 & 0.05 & 0.36 & 0.24 \\
\hline UGEP26 & 0.89 & 3.0 & 0.20 & 0.17 & 0.50 & 0.16 & UGEP102 & 0.89 & 1.0 & 0.21 & 0.16 & 0.50 & 0.22 \\
\hline UGEP27 & 0.99 & 3.0 & 0.02 & 0.01 & 0.32 & 0.67 & UGEP58 & 0.99 & 3.0 & 0.02 & 0.01 & 0.32 & 0.67 \\
\hline UGEP28 & 0.96 & 4.0 & 0.08 & 0.07 & 0.38 & 0.16 & UGEP59 & 0.98 & 4.0 & 0.05 & 0.03 & 0.35 & 0.33 \\
\hline UGEP29 & 0.98 & 4.0 & 0.05 & 0.03 & 0.35 & 0.33 & UGEP60 & 0.85 & 2.0 & 0.27 & 0.24 & 0.56 & 0.10 \\
\hline UGEP31 & 0.88 & 2.0 & 0.23 & 0.17 & 0.52 & 0.25 & UGEP62 & 0.98 & 3.0 & 0.03 & 0.02 & 0.33 & 0.50 \\
\hline UGEP33 & 0.97 & 3.0 & 0.06 & 0.05 & 0.36 & 0.24 & UGEP64 & 0.98 & 4.0 & 0.03 & 0.02 & 0.33 & 0.50 \\
\hline UGEP34 & 0.97 & 3.0 & 0.05 & 0.02 & 0.35 & 0.57 & UGEP65 & 0.86 & 2.0 & 0.25 & 0.20 & 0.54 & 0.22 \\
\hline UGEP45 & 0.98 & 3.0 & 0.05 & 0.02 & 0.35 & 0.66 & UGEP66 & 0.99 & 2.0 & 0.02 & 0.01 & 0.32 & 0.67 \\
\hline UGEP46 & 0.97 & 2.0 & 0.05 & 0.04 & 0.35 & 0.28 & UGEP67 & 0.98 & 2.0 & 0.05 & 0.00 & 0.35 & 1.00 \\
\hline UGEP47 & 0.94 & 3.0 & 0.11 & 0.09 & 0.41 & 0.24 & UGEP104 & 0.90 & 3.0 & 0.19 & 0.17 & 0.48 & 0.09 \\
\hline UGEP50 & 0.96 & 3.0 & 0.08 & 0.05 & 0.38 & 0.34 & UGEP105 & 0.97 & 3.0 & 0.05 & 0.04 & 0.35 & 0.27 \\
\hline UGEP51 & 0.97 & 3.0 & 0.05 & 0.02 & 0.35 & 0.56 & UGEP106 & 0.89 & 2.0 & 0.20 & 0.20 & 0.50 & 0.00 \\
\hline UGEP52 & 0.86 & 2.0 & 0.25 & 0.23 & 0.54 & 0.09 & UGEP107 & 0.90 & 2.0 & 0.19 & 0.14 & 0.49 & 0.27 \\
\hline UGEP53 & 0.88 & 3.0 & 0.22 & 0.20 & 0.51 & 0.07 & UGEP108 & 0.87 & 3.0 & 0.24 & 0.17 & 0.53 & 0.28 \\
\hline UGEP54 & 0.94 & 4.0 & 0.11 & 0.07 & 0.41 & 0.38 & UGEP109 & 0.92 & 1.0 & 0.15 & 0.09 & 0.45 & 0.44 \\
\hline UGEP56 & 0.86 & 2.0 & 0.25 & 0.23 & 0.54 & 0.09 & UGEP110 & 0.87 & 2.0 & 0.24 & 0.23 & 0.53 & 0.05 \\
\hline UGEP57 & 0.96 & 4.0 & 0.07 & 0.05 & 0.37 & 0.21 & UGEP111 & 0.93 & 2.0 & 0.13 & 0.11 & 0.43 & 0.19 \\
\hline UGEP103 & 0.95 & 4.0 & 0.09 & 0.03 & 0.39 & 0.66 & Mean & 0.92 & 2.9 & 0.14 & 0.11 & 0.44 & 0.34 \\
\hline
\end{tabular}




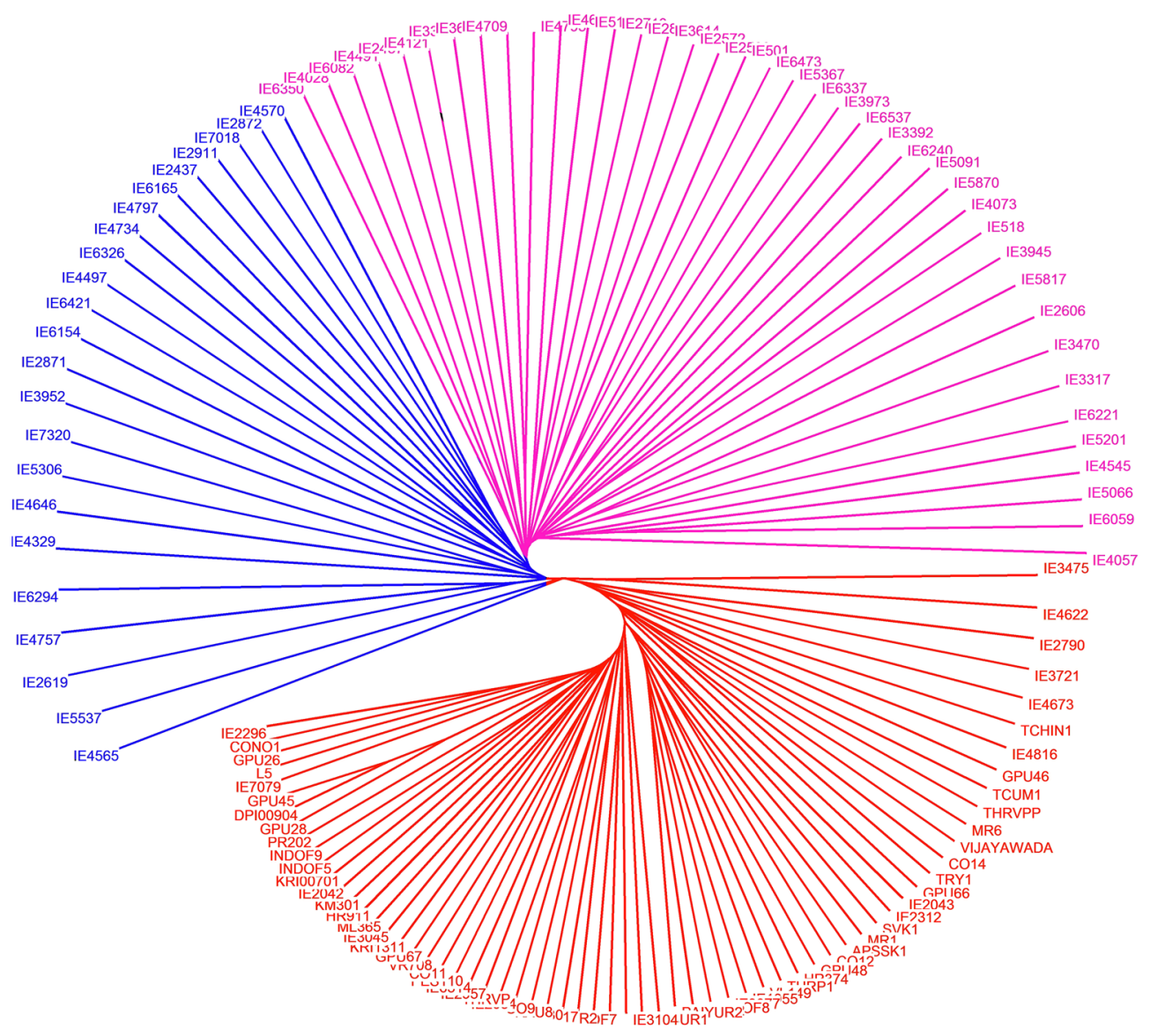

Fig. 1 UPGMA cluster analysis generated by Jaccard's similarity coefficient using 87 genomic SSR markers showing a genetic relationship in finger millet genotypes collected from various geographical regions of the world. Colors represent different subpopulations identified in structure analysis as shown in Figs. 3 and 4

Indian origin (close to first coordinate). Other 2 Indian genotypes GPU-46 and IE-4816 were dispersed in first coordinate; however the position of GPU-46 and IE-4816 were close to third coordinate. This result corresponded to dendogram and Jaccard's similarity coefficients analyses as GPU-28, GPU-45 and DPI009-04 were placed in the same $E c M C 1$. PCA scatter diagram showed that the jolliffe cut off value was 0.16809 and the first 65 principal components (PCs) with eigenvalues greater (5.049570.172131 ) than this cut-off value. PCs associated with the covariance matrix had eigenvalues greater in size than the average of all the eigenvalues showing that PCs were significant. The CCC value was 0.9216 which indicated that the cluster result was very good and acceptable to the genetic similarity matrix calculation.

\section{Population structure}

Structure analysis showed that the maximum $\Delta K$ value observed was $K=3$ which suggested that the 128 finger millet genotypes were broadly divided into three
SPs (SP1, SP2 and SP3) (Fig. 3). The genetic relationship among the SPs provided various confirmations for gene flow between SPs. This group also confirmed the Jaccard's similarity coefficient analysis which resulted in grouping of finger millet genotypes into three major clusters $(E c \mathrm{MC} 1-E c \mathrm{MC} 3)$. Indian genotypes were placed in first two SPs (SP1 and SP2) and non-Indian genotypes were placed in last two SPs (SP2 and SP3). This result confirmed that SP2 had both Indian and non-Indian genotypes and the results of the structure showed that all the three SPs had an admixture of alleles and no pure line was observed. Indian genotypes, VR-708, INDOF9, DPI009-04, IE-3077 and Paiyur-2 were $85-95 \%$ pure lines (Fig. 4). These genotypes were grouped in SP1 and SP2 and clustered at EcMC1 in UPGMA-Jaccard's similarity coefficient analysis.

\section{AMOVA analysis}

Hierarchical analysis of Indian and non-Indian finger millet genotypes' diversity was performed using AMOVA 


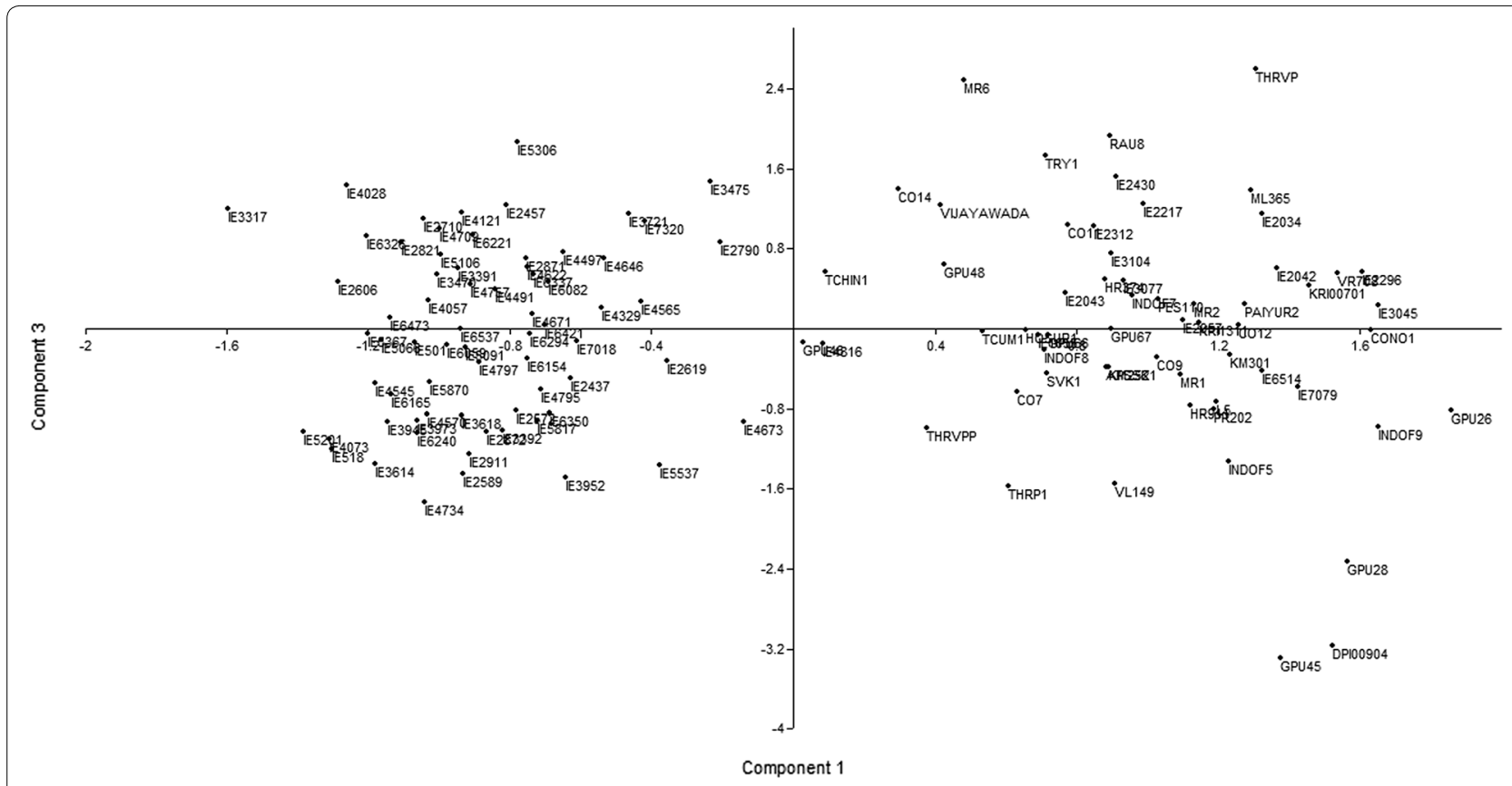

Fig. 2 PCA scatter diagram analysis showing the distributions of finger millet genotypes. Component 1 and 3 are the principal components of first and the third respectively

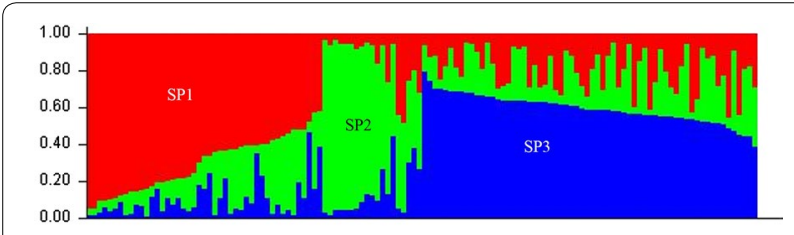

Fig. 3 The population structure analysis; the 128 finger millet genotypes were grouped into three subpopulations based on structure analysis

to know the variation in genotypes from various geographical regions, among populations and within populations. AMOVA analysis showed the degree of freedom (df) value among the various geographical regions, among populations and within populations as 9, 3 and 115 respectively. Sums of squared deviations' (SS) value among the various geographical regions, among populations and within populations were 588.72, 178.54 and 4539.23 respectively. The means of squared deviation (MS) values among the various geographical regions, among populations and within populations were 65.41, 59.51 and 39.47 respectively. Percentage of molecular variance among the various geographical regions was $4 \%$; among populations it was $3 \%$ and within populations it was 93 \% (Fig. 5). The Phi RT, Phi PR, Phi PT, Phi RT max, Phi 'RT, Phi PR max and Phi' PR values were $0.039,0.032,0.069,0.534,0.072,0.535$ and 0.060 respectively. Phi RT and Phi PT P value was 0.001 and Phi PR
$P$-value was 0.003 ; these values were less than 1 which confirmed the AMOVA results. The genotypic diversity value $(\mathrm{P})$ was highly significant $(p<0.001)$ at all the three hierarchical levels (among the various geographical regions, among populations and within populations). The highest value of genetic variation was observed among Indian populations (2722.43) and it was lower (31.0) in the Burundi and Nigerian populations. Among non-Indian genotypes, highest value of genetic variation (712.38) was found in Zimbabwean genotypes followed by Uganda genotypes (308.50). There was good correspondence between the Jaccard's similarity coefficient, PCA, population structure and the AMOVA in differentiating the finger millet genotypes into different clusters based on their geographical regions.

\section{Discussion}

\section{Finger millet genotypes}

The presented genetic relationships analyses are the first one based on 45 Southern Indian genotypes using 87 genomic SSR markers. PIC value, allele frequency, gene diversity and other indices of all polymorphic markers clearly demonstrated that SSRs could be successfully used for such studies. Our results are in agreement with results presented by others in finger millet as follows: 67 genotypes using 14 genomic SSR markers (Arya et al. 2013); 52 genotypes using 24 genomic SSR markers (Kumar et al. 2012), 79 genotypes using 45 genomic 


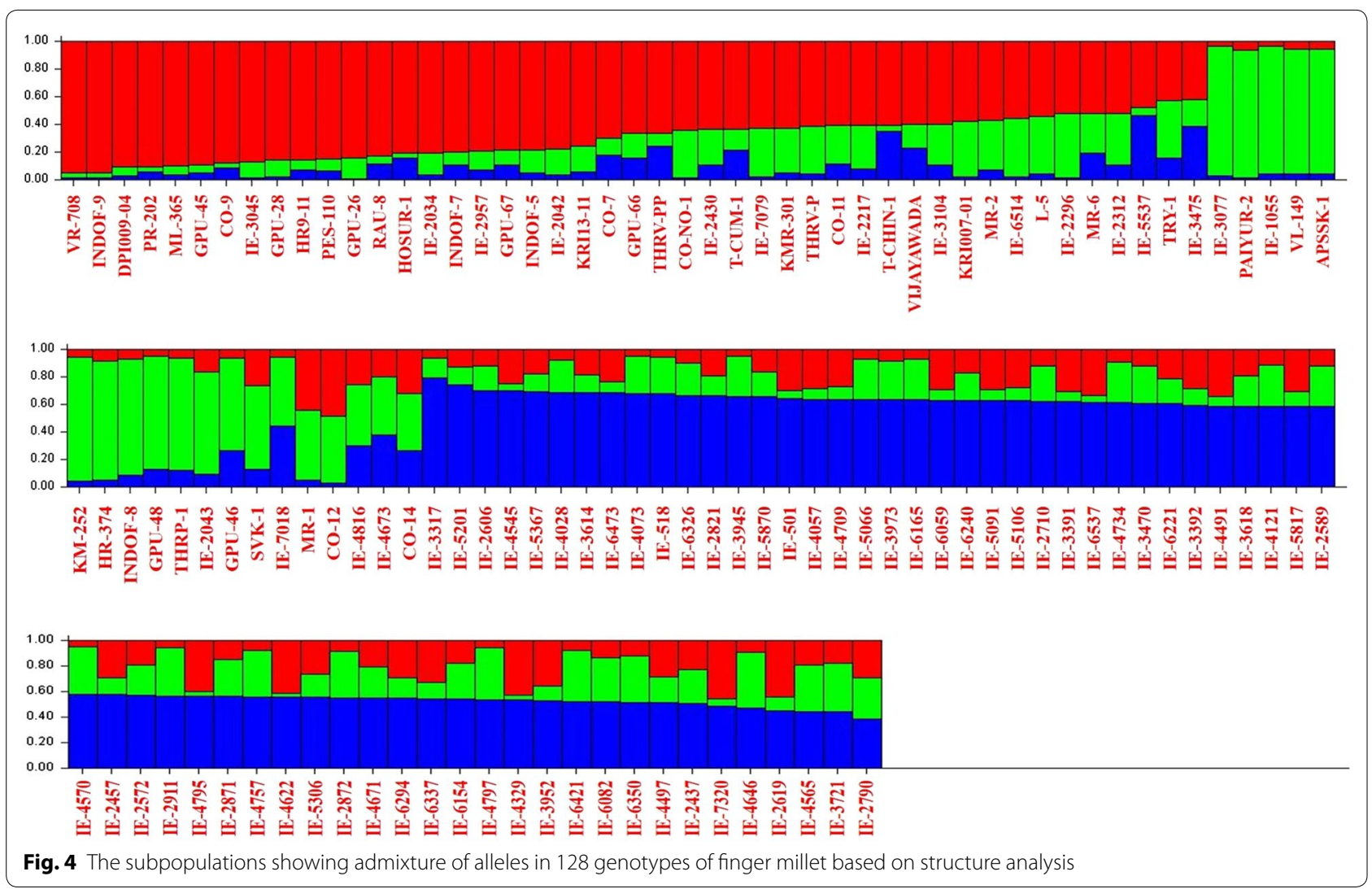

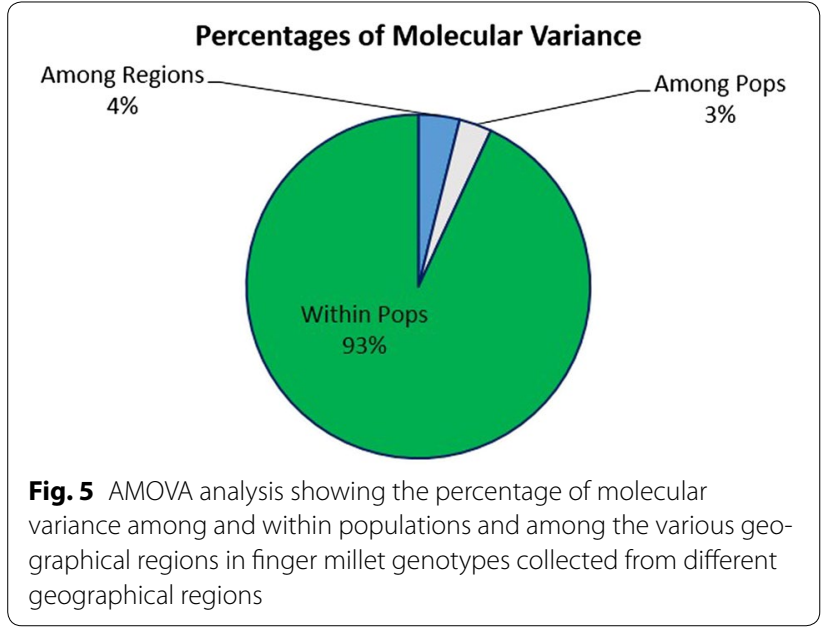

SSR markers (Dida et al. 2008), 83 genotypes using 10 genomic SSR markers (Panwar et al. 2010b).

\section{Genetic diversity analysis}

In general, use of SSR markers in millets to study the genetic diversity is a most appropriate technique providing useful molecular data when compared to the other marker systems and it has increased acceptance world-wide (Stich et al. 2010). In the present study SSR markers showed $73.80 \%$ polymorphism using 87 genomic SSR markers in 128 genotypes. However, out of 87 markers, only 72 markers $(82.75 \%$ ) were found to be polymorphic. It may be due to the fact that finger millet is a highly self-pollinating crop which might have caused low level of polymorphism in this study. This is in agreement with previous reports of Babu et al. (2014a, b) who reported that 35 (48\%) out of 74 genic SSR primers and 21 (46\%) out of 46 genomic SSR primers were found to be polymorphic in a study using 190 genotypes of finger millet.

In finger millet genotypes only a few reports are available on genetic diversity analysis using SSR markers with good amount of polymorphism. These are; $70.19 \%$ polymorphism in 83 genotypes using 10 genomic SSR markers (Panwar et al. 2010b); $66.6 \%$ polymorphism in 52 genotypes using 24 genomic SSR markers (Kumar et al. 2012), $68.23 \%$ polymorphism in 103 genotypes using 30 genic SSR markers (Nirgude et al. 2014). Similarly, Babu et al. (2014a, b) reported $72.22 \%$ polymorphism using 46 genomic SSR makers and $70.67 \%$ polymorphism using 74 genic SSR markers in 190 genotypes. In the present study we have detected highest polymorphism percentage ranging from 25.0 to $85.71 \%$ with an average of $59.94 \%$ 
polymorphism; this covered all the chromosomes in all genotypes. The highest polymorphism of $85.71 \%$ was detected with primer SSR10 and lowest polymorphism of $25.0 \%$ was detected with primer UGEP69.

In the present study, genomic SSR markers produced highest average of 1.06 alleles per Indian genotypes of finger millet. Similarly, Panwar et al. (2010b) and Arya et al. (2013) reported an average of 0.84 and 1.02 alleles respectively per finger millet genotype using SSR markers. Also, Babu et al. (2014b) reported an average of 0.7 allele per finger millet genotype using 74 genic SSR markers. The PIC values ranged from 0.32 to 0 . 64; average PIC value was 0.44 which showed the ability of genomic SSR markers to discriminate the Indian and non-Indian genotypes. This is in agreement with previous reports using SSR markers in finger millet (Babu et al. 2014b).

The gene diversity values were in the range of $0.02-0.35$ with an average value of 0.14 . This is in agreement with previous report by Babu et al. (2014a) based on genomic SSR markers in finger millet genotypes. However, based on genic SSRs, Nirgude et al. (2014) observed lower gene diversity values $(0.02-0.32)$ compared to our results. This low amount of gene diversity may be due to the lower number (15) of genic SSR markers they used. Babu et al. (2014b) observed higher amount of heterozygosity ranging from 0.00 to 1.00 with an average of 0.278 in 190 finger millet genotypes using 74 genic SSR markers. This may be due to the fact that lower number of alleles was produced by genic SSR markers. In the present study, we have observed lower heterozygosity value ranging from 0 to 0.26 with an average heterozygosity value of 0.11 , which was close to $100 \%$ homozygosity. The inbreeding coefficient value ranged from 0 to 1 and the average value was 0.34 . The SSRs which had heterozygosity value of 0 , showed highest inbreeding coefficient value of 1 . The gene diversity and heterozygosity present in Indian and non-Indian genotypes of finger millet showed that the genomic SSR markers used in the present study were more polymorphic. Similar results were obtained in 155 foxtail millet genotypes by Vetriventhan et al. (2013), using 72 genomic SSR markers where an average heterozygosity of 0.04 was recorded, which was close to $100 \%$ homozygosity.

The average similarity coefficient value was higher in Indian genotypes $(0.346)$ than those in non-Indian genotypes $(0.220)$. Also, similarity coefficient value was higher for those genotypes collected from Southern India with least similarity coefficient value for those from Zimbabwe, Nepal, Uganda and Kenya. This confirmed that the Indian genotypes might have originated from the same breeding population and non-Indian genotypes might have originated from different breeding population. Similarly, Arya et al. (2013) reported higher similarity coefficient value among finger millet genotypes collected from India and the least similarity coefficient value from genotypes of Africa using genomic SSR markers. Also, Bashir et al. (2015) detected higher similarity coefficient in pearl millet using SSR markers.

The bootstrap value was $100 \%$ in the final cluster node which confirmed that all genotypes were genetically diverse. This is in agreement with previous reports by Panwar et al. (2010b) in 83 finger millet genotypes collected from various regions of India and Africa. Similarly, Dida et al. (2008) analyzed 79 finger millet genotypes collected from Africa, Asia, Uganda and Kenya using genomic SSR markers which showed the bootstrap value of $100 \%$ in the final cluster node. Panwar et al. (2010b) reported that CCC value was 0.675 in 83 finger millet genotypes using SSR primers. However, in the present study, we have obtained highest CCC value of 0.9216 which is associated with acceptable genetic similarity matrix. Similarly, Ghasemi Ghehsareh et al. (2015) also obtained highest CCC value of 0.9968 in 53 genotypes representing eight species collected from Iran using microsatellite markers.

The affinities produced by PCA are generally in agreement with the results of the UPGMA cluster analysis. The first and third components axes accounted for $15.29 \%$ of the total variance. The genotypes were distributed according to their geographical regions and especially Indian genotypes were dispersed according to the local site of collection; this suggested that different sites in the PCA plot were good to estimate the genetic diversity. The affinities produced by PCA are generally in agreement with the results of the structure analysis. Similarly, Koehmstedt et al. (2010) observed that first two components of the PCA produced $24.8 \%$ of total variation using 15 SSR markers among a subset of 99 olive genotypes collected from the United States Department of Agriculture in Davis. Dossett et al. (2012) found that first three eigenvalues produced $9.6 \%$ of variance in 148 genotypes of blackcap using 21 SSR markers. In the present study, we also observed first three eigenvalues of $7.94 \%$.

The AMOVA analysis showed that the percentages of molecular variance among the various geographical regions, among populations and within populations were 4,3 and $93 \%$ respectively. Similarly, Babu et al. (2014b) reported molecular variance only within populations $(73 \%)$ and among populations $(27 \%)$ in 190 finger millet genotypes using 74 genic SSR markers. The present study is the first and detailed report on genetic diversity analysis of 128 finger millet genotypes based on their geographical regions using genomic SSR markers. AMOVA analysis showed significant differences between the genotypes and also produced greater percentage of molecular variance among the geographical regions, 
among populations and within populations. This was due to the self-pollinating nature of finger millet. It was also proved by population structure analysis that these populations were genetically isolated from each other. There was good correspondence between the AMOVA and the population structure in differentiating the finger millet genotypes into different clusters.

Structure analysis showed maximum $\mathrm{D} K$ value of $K=3$; 128 genotypes were divided into three subpopulations (SP1, SP2 and SP3). In the present study using SSR markers all subpopulations had an admixture of alleles and no pure line was observed. This is in agreement with previous report of Dida et al. (2008) who observed an admixture of alleles with African and Asian alleles of finger millet genotypes using 45 SSR markers. Similarly Babu et al. (2014b) also identified four subpopulations among 190 finger millet genotypes using 74 genic SSR makers with an admixture of alleles from other populations and no pure line was observed. SSR marker system has been found to be superior over other markers like RFLP, RAPD, ISSR, and AFLP. In the present study, we have observed that Paiyur-2 had only $95 \%$ purity this may be due to locus specific alleles produced by SSR markers; this was not obtained in previous study using RAPD markers (Ramakrishnan et al. 2015). This study helped to predict the important genotypes with putative agronomic traits. We found that Paiyur-2 is a high yielding genotype cultivated in Southern India and this genotype was found to be $95 \%$ pure line. Population structure corresponded to PCA, AMOVA and Jaccard's similarity coefficient. Similarly Khadari et al. (2003), Hazarika et al. (2014) and Khan et al. (2014) chose only SSR markers as the markers of choice for breeding research, because of their locus specificity and variability, ease to use, accessibility of detection, reproducibility and data exchange.

In conclusion, we have confirmed that all 128 genotypes were genetically diverse and were clustered into three subpopulations based on their geographic region of origin. Data generated through this study may be utilized for mapping of any important agronomical trait for breeding programes to improve the finger millet.

\begin{abstract}
Abbreviations
NBPGR: National bureau of plant genetic resources; ICRISAT: International crops research institute for the semi-arid-tropics; AICMMP: All India coordinated minor millet project; SSR: Simple sequence repeats; EST-SSR: Expressed sequence tag- simple sequence repeats; PIC: Polymorphic information content; UPGMA: Unweighted pair-group method with arithmetic average; AMOVA: Analysis of the molecular variation; CCC: Cophenetic correlation coefficient; PCA: Principal component analysis; SPs: Subpopulations; MCMC: Markov Chain Monte Carlo.
\end{abstract}

\section{Authors' contributions}

MR carried out the study and drafted the manuscript. SAC was involved in software analysis and manuscript preparation. SI participated in the design of the study, interpretation of data and manuscript preparation. VD was involved in the design of study and analysis and interpretation of data. ND conceived of the study and helped. All authors read and approved the final manuscript.

\section{Author details}

1 Division of Plant Biotechnology, Entomology Research Institute, Loyola College, Chennai 600 034, India. ${ }^{2}$ Faculty of Biological Sciences, Centre for Plant Sciences and School of Molecular and Cellular Biology, University of Leeds, Leeds LS2 9JT, UK. ${ }^{3}$ Department of Botany and Microbiology, Addiriyah Chair for Environmental Studies, College of Science, King Saud University, P.O.Box. 2455, Riyadh 11451, Kingdom of Saudi Arabia. ${ }^{4}$ Visiting Professor Program, Deanship of Scientific Research, College of Science, King Saud University, P.O.Box. 2455, Riyadh 11451, Kingdom of Saudi Arabia.

\section{Acknowledgements}

We thank the Department of Biotechnology, New Delhi, for providing financial support (BT/PR15011/AGR/02/772/2010). We thank the Deanship of Scientific Research, College of science, King Saud University, Kingdom of Saudi Arabia, for providing financial support through the Research Group project No RGP-VPP-213. We thank ICRISAT, UAS (GKVK) and TNAU for supplying the finger millet seeds. Also we thank the mentors of this project, Dr. P. Kadirvel, Senior Scientist, DOR, ICAR, Hyderabad-500030-India, Prof. P. Balasubramanian, Centre for Plant Molecular Biology, TNAU, Coimbatore-641003-India, Dr. T.C. Santiago, Emeritus Scientist and Dr. Joseph Sahaya rajan, CIBA, ICAR, Chennai600028-India, for their encouragement. We thank Mr. Vanlalhruaia, Division of Ethanopharmocology, Entomology research Institute, Loyola College, for image processing. Also we thank the reviewers for their valuable comments and suggestions to improve the article quality.

\section{Competing interests}

The authors declare that they have no competing interests.

Received: 13 August 2015 Accepted: 16 December 2015

Published online: 11 February 2016

\section{References}

Arya L, Verma M, Gupta VK, Seetharam A (2013) Use of genomic and genic SSR markers for assessing genetic diversity and population structure in Indian and African finger millet (Eleusine coracana (L.) Gaertn.) germplasm. Plant Syst Evol 299:1395-1401

Babu BK, Agrawal PK, Pandey D, Jaiswal JP, Kumar A (2014a) Association mapping of agro-morphological characters among the global collection of finger millet genotypes using genomic SSR markers. Mol Biol Rep 41:5287-5297

Babu BK, Agrawal PK, Pandey D, Kumar A (2014b) Comparative genomics and association mapping approaches for opaque2 modifier genes in finger millet accessions using genic, genomic and candidate gene-based simple sequence repeat markers. Mol Breed 34:1261-1279

Bashir EA, Ali A, Ali A, Mohamed E, Melchinger A, Parzies H, Haussmann BG (2015) Genetic diversity of Sudanese pearl millet (Pennisetum glaucum (L.) R. Br.) landraces as revealed by SSR markers, and relationship between genetic and agro-morphological diversity. Genet Resour Crop Evol 62:579-591

Cho YG, Ishii T, Temnykh S, Chen X, Lipovich L, McCouch SR, Park WD, Ayres $\mathrm{N}$, Cartinhour S (2000) Diversity of microsatellites derived from genomic libraries and GenBank sequences in rice (Oryza sativa L.). Theor Appl Genet 100:713-722

Cho Y-I, Chung J-W, Lee G-A, Ma K-H, Dixit A, Gwag J-G, Park Y-J (2010) Development and characterization of twenty-five new polymorphic microsatellite markers in proso millet (Panicum miliaceum L.). Genes Genom 32:267-273

Dida MM, Wanyera N, Harrison Dunn ML, Bennetzen JL, Devos KM (2008) Population Structure and Diversity in Finger Millet (Eleusine coracana) Germplasm. Tropical Plant Biology 1:131-141

Dossett M, Bassil NV, Lewers KS, Finn CE (2012) Genetic diversity in wild and cultivated black raspberry (Rubus occidentalis L.) evaluated by simple sequence repeat markers. Genet Resour Crop Evol 59:1849-1865

Egbadzor K, Ofori K, Yeboah M, Aboagye L, Opoku-Agyeman M, Danquah E, Offei S (2014) Diversity in 113 cowpea [Vigna unguiculata (L) Walp] accessions assessed with 458 SNP markers. SpringerPlus 3:1-15

Evanno G, Regnaut S, Goudet J (2005) Detecting the number of clusters of individuals using the software structure: a simulation study. Mol Ecol 14:2611-2620 
Ghasemi Ghehsareh M, Salehi H, Khosh-Khui M, Niazi A (2015) Application of ISSR markers to analyze molecular relationships in Iranian jasmine (Jasminum spp.) accessions. Mol Biotechnol 57:65-74

Goron TL, Raizada MN (2015) Genetic diversity and genomic resources available for the small millet crops to accelerate a New Green Revolution. Front Plant Sci 6:157

Hammer $\varnothing$, Harper D, Ryan P (2001) PAST: paleontological statistics software package for education and data analysis. Palaeontol Electron 4:9

Hazarika TK, Hazarika BN, Shukla AC (2014) Genetic variability and phylogenetic relationships studies of genus Citrus L. with the application of molecular markers. Genet Resour Crop Evol 1-14

Hua W, Zhang X, Zhu J, Shang Y, Wang J, Jia Q, Li C, Yang J (2015) A study of genetic diversity of colored barley (Hordeum vulgare L.) using SSR markers. Genet Resour Crop Evol 62:395-406

Jaccard P (1908) Nouvelles researches sur la distribution florale. Bull Soc Vaudoise Sci Natl 44:223-270

Jolliffe IT (2002) Principal component analysis, 2nd edn. Springer, New York

Kanatti A, Rai K, Radhika K, Govindaraj M, Sahrawat K, Rao A (2014) Grain iron and zinc density in pearl millet: combining ability, heterosis and association with grain yield and grain size. SpringerPlus 3:1-12

Karandikar R (2006) On the Markov Chain Monte Carlo (MCMC) method. Sadhana 31:81-84

Khadari B, Breton C, Moutier N, Roger JP, Besnard G, Berville A, Dosba F (2003) The use of molecular markers for germplasm management in a French olive collection. Theor Appl Genet 106:521-529

Khan MK, Pandey A, Choudhary S, Hakki E, Akkaya M, Thomas G (2014) From RFLP to DArT: molecular tools for wheat (Triticum spp.) diversity analysis. Genet Resour Crop Evol 61:1001-1032

Kim E, Sa K, Park K-C, Lee J (2012) Study of genetic diversity and relationships among accessions of foxtail millet [Setaria italica (L.) P. Beauv.] in Korea, China, and Pakistan using SSR markers. Genes Genom 34:529-538

Koehmstedt AM, Aradhya MK, Soleri D, Smith JL, Polito VS (2010) Molecular characterization of genetic diversity, structure, and differentiation in the olive (Olea europaea L.) germplasm collection of the United States Department of Agriculture. Genet Resour Crop Evol 58:519-531

Kumar A, Sharma N, Panwar P, Gupta A (2012) Use of SSR, RAPD markers and protein profiles based analysis to differentiate Eleusine coracana genotypes differing in their protein content. Mol Biol Rep 39:4949-4960

Liu K, Muse M (2005) Power Marker: an integrated analysis environment for genetic marker analysis. Bioinformatics 21:2128-2129

Nirgude M, Babu BK, Shambhavi Y, Singh UM, Upadhyaya HD, Kumar A (2014) Development and molecular characterization of genic molecular markers for grain protein and calcium content in finger millet (Eleusine coracana (L.) Gaertn.). Mol Biol Rep 41:1189-1200

Panwar P, Nath M, Yadav VK, Kumar A (2010a) Comparative evaluation of genetic diversity using RAPD, SSR and cytochrome P450 gene based markers with respect to calcium content in finger millet (Eleusine coracana L. Gaertn.). J Genet 89:121-133
Panwar P, Saini RK, Sharma N, Yadav D, Kumar A (2010b) Efficiency of RAPD, SSR and cytochrome P450 gene based markers in accessing genetic variability amongst finger millet (Eleusine coracana) accessions. Mol Biol Rep 37:4075-4082

Peakall R, Smouse PE (2012) GenAlEx 6.5: genetic analysis in Excel. Population genetic software for teaching and research - an update. Bioinformatics 28:2537-2589

Pritchard JK, Stephens M, Donnelly P (2000) Inference of population structure using multilocus genotype data. Genetics 155:945-959

Qin Y, Kim S-M, Sohn J-K (2009) Genetic analysis and QTL mapping for grain chalkiness characteristics of brown rice (Oryza sativa L.). Genes Genom 31:155-164

Ramakrishnan M, Ceasar SA, Duraipandiyan V, Al-Dhabi NA, Ignacimuthu S (2015) Using molecular markers to assess the genetic diversity and population structure of finger millet (Eleusine coracana (L.) Gaertn.) from various geographical regions. Genet Resour Crop Evol. doi:10.1007/ s10722-015-0255-1

Ramasamy R, Ramasamy S, Bindroo B, Naik V (2014) STRUCTURE PLOT: a program for drawing elegant STRUCTURE bar plots in user friendly interface. SpringerPlus 3:1-3

Scott KD, Eggler P, Seaton G, Rossetto M, Ablett EM, Lee LS, Henry RJ (2000) Analysis of SSRs derived from grape ESTs. Theor Appl Genet 100:723-726

Sharma M, Verma R, Singh A, Batra A (2014) Assessment of clonal fidelity of Tylophora indica (Burm. f.) Merrill "in vitro" plantlets by ISSR molecular markers. SpringerPlus 3:1-9

Stich B, Haussmann B, Pasam R, Bhosale S, Hash CT, Melchinger A, Parzies H (2010) Patterns of molecular and phenotypic diversity in pearl millet [Pennisetum glaucum (L.) R. Br.] from West and Central Africa and their relation to geographical and environmental parameters. BMC Plant Biol 10:216

Vetriventhan M, Upadhyaya HD, Anandakumar CR, Senthilvel S, Varshney RK, Parzies HK (2013) Population structure and linkage disequilibrium of ICRISAT foxtail millet (Setaria italica (L.) P. Beauv.) core collection. Euphytica 196:423-435

Wang YW, Samuels TD, Wu YQ (2011) Development of 1030 genomic SSR markers in switchgrass. Theor Appl Genet 122:677-686

Yang L, Fu S, Khan M, Zeng W, Fu J (2013) Molecular cloning and development of RAPD-SCAR markers for Dimocarpus longan variety authentication. SpringerPlus 2:1-8

Zhao W, Lee G-A, Kwon S-W, Ma K-H, Lee M-C, Park Y-J (2012) Development and use of novel SSR markers for molecular genetic diversity in Italian millet (Setaria italica L.). Genes Genom 34:51-57

\section{Submit your manuscript to a SpringerOpen ${ }^{\circ}$ journal and benefit from:}

- Convenient online submission

- Rigorous peer review

- Immediate publication on acceptance

- Open access: articles freely available online

- High visibility within the field

- Retaining the copyright to your article

Submit your next manuscript at $>$ springeropen.com 